\title{
An investigation of the role of background music in IVWs for learning
}

\author{
Debbie Richards $^{\mathrm{a} *}$, Eric Fassbender ${ }^{\mathrm{a}}$, Ayse Bilgin ${ }^{\mathrm{b}}$ and William Forde Thompson ${ }^{\mathrm{c}}$ \\ ${ }^{a}$ Department of Computing, Macquarie University, Sydney, Australia; ${ }^{b}$ Department of Statistics, \\ Macquarie University, Sydney, Australia; ${ }^{c}$ Department of Psychology, Macquarie University, \\ Sydney, Australia
}

(Received 31 March 2008; final version received 8 September 2008)

\begin{abstract}
Empirical evidence is needed to corroborate the intuitions of gamers and game developers in understanding the benefits of Immersive Virtual Worlds (IVWs) as a learning environment and the role that music plays within these environments. We report an investigation to determine if background music of the genre typically found in computer-based role-playing games has an effect on learning in a computer-animated history lesson about the Macquarie Lighthouse within an IVW. In Experiment 1, music stimuli were created from four different computer game soundtracks. Seventy-two undergraduate students watched the presentation and completed a survey including biographical details, questions on the historical material presented and questions relating to their perceived level of immersion. While the tempo and pitch of the music was unrelated to learning, music conditions resulted in a higher number of accurately remembered facts than the no music condition. One soundtrack showed a statistically significant improvement in memorisation of facts over other music conditions. Also an interaction between the levels of perceived immersion and ability to accurately remember facts was observed. Experiment 2, involving 48 undergraduate students, further investigated the effect of music, sense of immersion and how different display systems affect memory for facts.
\end{abstract}

Keywords: immersive virtual worlds; learning; music

\section{Introduction}

Technology does not offer an automatic solution to the various problems experienced in education. From an economic point of view it is often difficult to find any evidence of the benefits of technology (Landauer 1995). However, when there is "a good fit between a particular learning situation and specific technical solution" striking positive results can be found (Draper, Cargill, and Cutts 2002, 16). The benefits of using computer games which allow students (children in these particular cases) to learn as they play are demonstrated in games like 'Kahootz' (http:/www.kahootz.com/), 'Where in the World is Carmen San Diego?' (http://www.learningcompany.com/) and 'Zoombinies' (http://www.learningcompany.com/ ) which require and train problem-solving abilities. Going a step beyond such computer games are immersive virtual worlds (IVWs) which allow students to engage in experiential learning through role-playing, and has been recognised as a useful means of learning new skills, practicing problem-solving and gaining experience. Mason and Mitroff state that "stories, drama, role-plays, art, graphics, one-to-one contact and group discussions may be more effective in some information contexts [... over] the language of abstract symbols and 'hard' data"

*Corresponding author. Email: richards@ics.mq.edu.au 
$(1973,484)$. IVWs offer a means to facilitate distributed learning that is not restricted to a specific physical location or time. Further, the costs involved in bringing people together and setting up a convenient and simulated or real environment are reduced. In many cases, such as training fire fighters to put out a house fire (for example, St. Julian and Shaw 2003), an IVW makes possible something that is prohibitively expensive, dangerous or undesirable in the natural world.

To date, the IVW field has been driven by the entertainment industry rather than educationalists. The result has been that while examples of successful and useful systems can be found, there is often little understanding and usually no theory behind whether or why something does or does not assist learning. Much of the use of games and IVWs in education is based on assumptions such as that interactivity will produce better learning and that immersion will produce greater engagement and lead to better learning. But the evidence for such assumptions is not clear. For example, in our earlier experiments we found that interactivity does not always lead to improved learning (Richards 2006). Despite the lack of theory and empirical evidence for the value or role that music plays (a discussion of relevant issues can be found in Zehnder and Lipscomb 2006), we nevertheless find music within most, if not all, game environments. We can conclude that game developers intuitively believe that music is a desirable feature even if its effects are not understood. In this paper we report our investigations of music within an IVW and its effect on learning while considering what others have found in related areas.

\section{Literature review}

One of the most well-known studies concerning music and intelligence produced the socalled 'Mozart Effect' (Rauscher, Shaw, and Ky 1993). This study investigated the effect of listening to classical music before subjects performed a spatial reasoning test. They found that 36 college students who listened to 10 minutes of Mozart's Sonata for Two Pianos in D Major, K448, scored 8-9 points higher in a subsequent spatial ability IQ test, compared to when they listened to a relaxation tape or to no audio stimulus at all. The results have been heavily discussed in the literature and other researchers have tried to replicate the results, some with success (Rideout and Dougherty 1998) and others not (McKelvie and Low 2002). The current view is that such effects of music on spatial-temporal processing are related to temporary changes in mood and arousal levels that arise from music listening (Husain, Thompson, and Schellenberg 2002; Thompson, Schellenberg, and Husain 2001). Along these lines, Bulgarian psychologist Lozanov (1992) recommended several specific musical pieces that make listeners open to suggestion and put the listener into a state of mind that is beneficial for learning foreign languages (Lozanov 1988).

A somewhat different study was conducted by McFarland and Kennison (1988) who found that subjects who had to solve a tactual maze task with either their right or left hand performed badly when they were listening to music with the ear that was on the same side of the body as the hand they were performing the task with, due to 'intrahemispheric competition'. Typical of many music studies, under some circumstances music improved performance and at other times worsened performance. Another study linking music and learning at the psycho-physiological level was conducted by Dryden and Vos (2001) who found that for learning, the 'relaxed alertness' state of mind known as the alpha state was the most beneficial.

Much of the research on the role of music on learning attributes an indirect link between the two. For example, Vester (1975) says that we learn and remember more, the more senses are included in the learning process. We could thus conclude that when we add music to 
visual and/or other aural stimuli, greater learning is possible. Davies states that "Music's power to evoke emotions also enhances learning", and goes on to say that "heightened involvement creates a stronger neural connection, which in turn makes it easier to remember information" $(2000,149)$. We believe that this is even truer if the visual and the aural senses are combined. In our earlier work (Fassbender and Heiden 2006), we found evidence for the benefit of the inclusion of the visual sense on learning. Our study included 'The Virtual Memory Palace', a 3D environment which offers the possibility to freely explore and utilise a virtual three-dimensional architectural representation of an ancient Greek (and formerly purely mental) memory technique.

Two areas of research particularly relevant to our study which we discuss further in the next section, are the work by Boltz, Schulkind, and Kantra (1991) on mood congruence in film music, and the work by Thompson and colleagues on the role of pitch and tempo on mood (Thompson, Schellenberg, and Husain 2001; Husain, Thompson, and Schellenberg 2002).

Boltz, Schulkind, and Kantra (1991) investigated the effects of background music on the remembering of filmed events and found that participants remembered visual information (i.e. movie scenes) better if such information was accompanied by mood-congruent music. Further experiments showed that mood-congruent pairings of video and audio are jointly encoded into the cognitive system (Boltz 2004). In their experiments, Thompson, Schellenberg, and Husain (2001) found that participants performed significantly better in an associated paper-folding and cutting task when they listened to a 'pleasant and energetic' Mozart piece in major key as opposed to a second group who listened to a 'slow, sad' Albioni piece in minor key. They see that the 'Mozart Effect' is more likely to be a result of mood and arousal, and conclude that:

It is possible, then, that the Mozart effect has little to do with Mozart in particular or with music in general. Rather, it may represent an example of enhanced performance caused by manipulation of arousal or mood. (Thompson, Schellenberg, and Husain 2001, 248)

\section{Research question}

Looking at the literature, there seem to be contradictory opinions and inconclusive evidence about the effect of music on learning. In our investigations we aim to explore whether music increases learning in an IVW. Our particular focus is on memorisation of facts. Our goal is to contribute insights and rigour to the study and practice of pedagogy via IVWs and provide a basis for theory development in the field. To this end, we have designed an IVW which serves a dual function: to provide a learning environment for the history of the Macquarie Lighthouse as well as an experimental environment within which our investigations can be made.

It is commonly observed that players frequently become completely occupied within an IVW and their attention is so intensely focused on the fantasy world in which they are roaming about, that almost nothing can disturb them and get them out of these worlds and back into reality. One explanation for this sense of engagement could be Csikszentmihalyi's 'Flow' theory (1990) which involves the "feeling of complete and energized focus in an activity, with a high level of enjoyment and fulfilment" (Chen 2007, 31). Another explanation could be that "video game play is an activity which lies in the domain of intrinsic motivation" (Holt 2000, 9). Furthermore, music seems to have an effect on motivation. In an unpublished doctoral thesis by Weisskoff (1981) "students who received the music condition scored significantly higher with regard to continuing motivation" (cited in Eady 
and Wilson 2004, 243) when reporting about a study of children receiving language lessons - with or without background music.

From our experience and observation, and in line with Zehnder and Lipscomb (2006), we believe that music contributes to the sense of total immersion within an IVW. Consequently, the aim of our research is to examine effects of music on memory for events and facts that are learned during game experiences and to explore the significance of music for feelings of immersion in game-like virtual-immersive environments. In our study we consider the potential effects of background music on the perception and memory of events and facts experienced in an IVW. As music is multidimensional, in our initial experiments we have narrowed our focus to the effect of pitch and tempo of music based on their powerful effects on emotion and mood (Thompson, Schellenberg, and Husain 2001; Ilie and Thompson 2006; Boltz 2004; Boltz, Schulkind, and Kantra 1991). Consequently, our research question asks how changes in pitch and tempo of soundtracks of computer-based role-playing games affect the memory for the historical facts delivered by an avatar (virtual persona) in the IVW.

\section{The Macquarie Lighthouse history lesson IVW}

As a precursor to answering the above research question it was necessary to create an IVW. To provide such a learning environment we chose to create a computer-animated history lesson presenting the history of the Macquarie Lighthouse, the first lighthouse of Australia and the southern hemisphere. Despite this importance to Australian settlement, the history of the Macquarie Lighthouse is not widely known and is thus an ideal topic for evaluating learning of historical facts. Since the effect of interactivity on learning was not part of this study, we created a non-interactive computer-animated history lesson to minimise the number of variables and provide greater control. Even though the course was non-interactive, it was created to look and feel like a typical conversation with an avatar in a fully featured computer role-playing game and IVW. During the computer-animated history lesson, numerous facts about the history of the lighthouse, including dates, names of people, ships, equipment and places are presented. Twenty-nine questions were asked of participants following the presentation to determine how well they recalled the information given.

To create the Macquarie Lighthouse IVW, a game mod (the technical term used to refer to a modification of the original application) was developed with 'The Elder Scrolls Construction Set' (TESCS) (http://www.elderscrolls.com/downloads/updates_utilities.htm). TESCS is an expansion for the game 'Elder Scrolls 4 - Oblivion' (Bethesda Softworks: http:/ /www.elderscrolls.com/home/home.htm) which allows the creation of complete virtual scenarios and supplies the developer with the necessary tools to design a virtual world in his or her own style. In the first step, the landscape surrounding the Macquarie Lighthouse was modelled in TESCS. To achieve a high level of authenticity, the landscape was modelled by means of an accurate land survey supplied by the Sydney Harbour Federation Trust. Afterwards, a 3D model of the Macquarie Lighthouse was created with 3D Studio Max (http:// www.autodesk.com) and then imported into TESCS (Figure 1). The avatar in the foreground is a fictitious descendant of the first lighthouse keeper, and delivers the history of the lighthouse to the users.

Six different versions of this history lesson were produced using different background musical stimuli, which will be explained in more detail later. The computer-animated history lesson was displayed by means of a Cave-like display system called a 'Cone' to increase the level of visual immersion. Figure 2 shows a pilot-tester who is watching the video narration and listening to the avatar. 


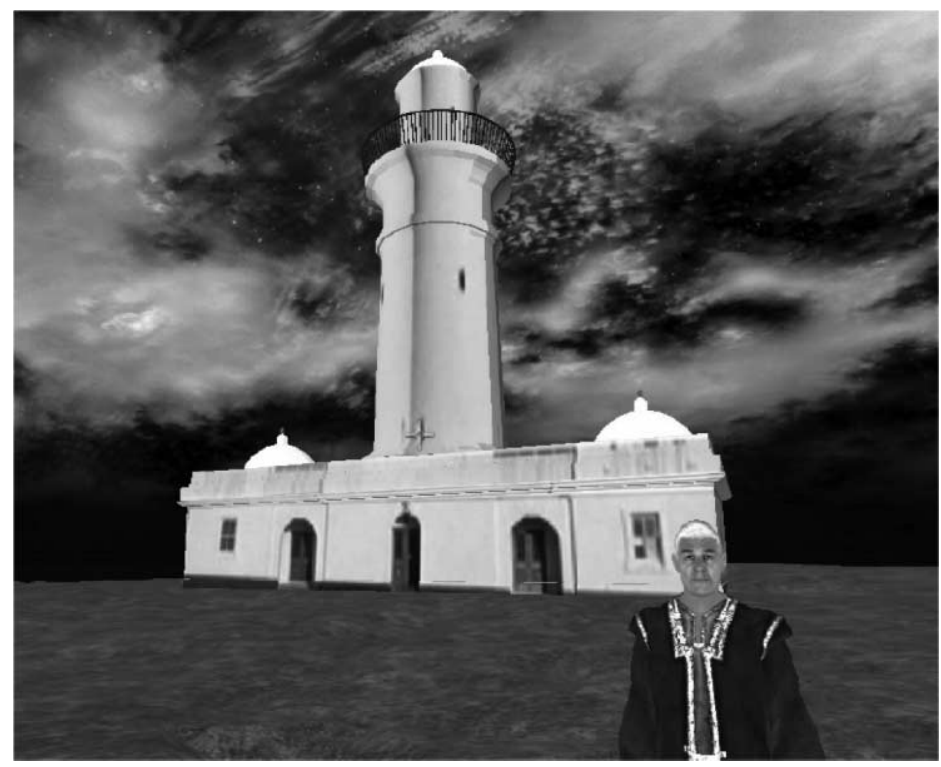

Figure 1. A 3D model of the Macquarie Lighthouse together with a virtual Avatar who is explaining the history of the lighthouse to the users.

The Cone-display system consists of three projectors which display the virtual world onto a semi-cylindrical screen canvas. The user is positioned slightly off centre towards the canvas to allow a $160^{\circ}$ field of view (FOV) which simulates almost the maximum of $180^{\circ}$ of the natural human FOV. Through this setup the virtual reality occupies most of the user's visual sense and the user gets the impression that the world displayed on the screen canvas is almost real; he or she feels immersed in this virtual reality.

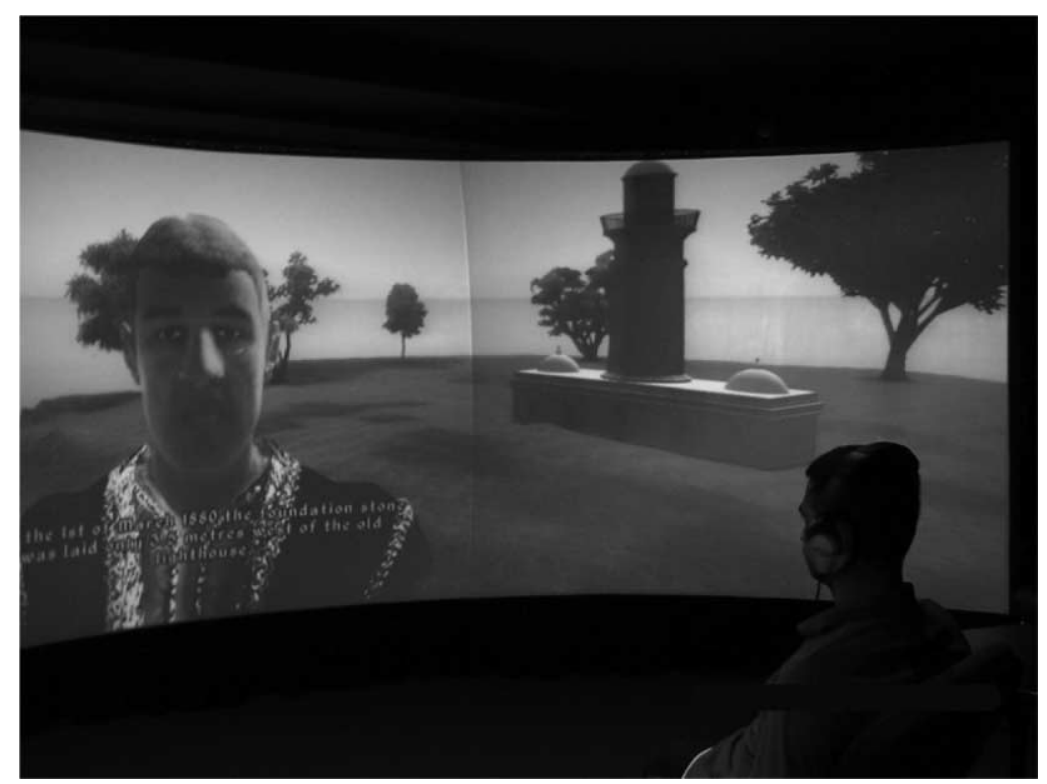

Figure 2. A pilot-tester watching and listening to the 3D narration about the history of the Macquarie Lighthouse. 


\section{Methodology - stimuli and measures}

In order to gain a somewhat representative sample of the computer role-playing game genre, the soundtracks from four contemporary computer role-playing games, namely 'Oblivion' (http://www.elderscrolls.com), 'Baldur's Gate' (http://www.bioware.com/games/baldurs_ gate/), 'World of Warcraft' (http://www.worldofwarcraft.com) and 'Icewind Dale' (http:// www.blackisle.com/ or http://www.mobygames.com/game/icewind-dale), were selected for manipulation. From each soundtrack of each game three musical pieces were pre-selected. These three pre-selected pieces were then presented to a group of six pilot-testers who were recruited from the Department of Computing at Macquarie University. All of the pilottesters have extensive experience with computer role-playing games and the associated soundtracks. The pilot-testers were asked to rank the musical pieces on a scale from 1 to 5 as being: (a) not representative (1) or representative (5) for the role-playing game genre; and (b) not suitable (1) or suitable (5) for the $3 \mathrm{D}$ animated video presentation. The musical pieces that scored the highest overall value in both categories were then selected for further experiments.

\section{Tempo and pitch manipulations}

As discussed earlier, two musical attributes that have powerful effects on emotion and mood (which in consequence might have an effect on learning and immersion) are pitch and tempo (Husain, Thompson, and Schellenberg 2002; Thompson, Schellenberg, and Husain 2001). Four musical pieces (Oblivion, Baldur's Gate, World of Warcraft, Icewind Dale) were selected and manipulated in tempo and pitch, based on work by Ilie and Thompson (2006). Tempo was reduced/increased by $21 \% / 26 \%$ and pitch was lowered/increased by two semitones. The manipulations were accomplished using a Macbook Pro in Ableton Live 6.0.7. Ableton Live is a professional DAW (Digital Audio Workstation) software, which allows convenient and high quality tempo and pitch manipulations. Resulting pieces were then presented to three music experts from the Department of Contemporary Music Studies at Macquarie University. None of the experts found irritating distortions or could identify the manipulations as such. The three possible tempos (slow, medium/original, fast) and three possible pitches (low, medium/original and high) resulted in nine possible combinations. For reasons of practicality (size of participant pool, available laboratory time, etc.) it was decided to test only five of the nine possibilities: Condition 1: S/L - Slow Tempo/Low Pitch; Condition 3: F/L - Fast Tempo/Low Pitch; Condition 5: M/M - Medium Tempo/ Medium Pitch (tempo and pitch unaltered); Condition 7: $\mathrm{S} / \mathrm{H}$ - Slow Tempo/High Pitch; Condition 9: F/H - Fast Tempo/High Pitch. Each of the five manipulations was performed on the four different musical pieces, indicating that all musical pieces received the same tempo/pitch manipulation. A control group (Condition 10) was assigned to the 'No Music' condition and during the $3 \mathrm{D}$ history course they only listened to the voice of the avatar without any musical background.

\section{Experiment 1}

A total of 72 undergraduate students (45 female, 27 male) from 19 to 56 years old (mean 24.2 years of age) were recruited by advertisement on campus and from introductory statistics and computing classes at Macquarie University, Sydney. They were allocated into six experimental conditions; therefore each experimental condition had 12 participants. Fifteen participants indicated English as their first language while 57 participants answered that English was their 'second or other' language. 
The experiment consisted of three stages:

- Stage 1 - a short pre-experiment biographical questionnaire (e.g. age, gender, first language, etc.).

- Stage 2-an 11:38 minute video narration of the history course. Participants sat in the centre of the display system where they watched and listened to the stimuli wearing high quality noise-cancelling stereo headphones.

- Stage 3 - a post-experiment set of questionnaires:

- 29 questions about the history of the Macquarie Lighthouse as covered in the video narration multiple-choice questionnaire about facts that they just learned in the history lesson (e.g., what was the name of the first lighthouse keeper, in which year was the first lighthouse built, how many people were stationed at the lighthouse, etc.).

- 27 questions about the immersiveness of the video narration (e.g., how well were you concentrating, how much did you lose track of time).

- 6 questions about participants' prior experience with music and computer/ console games (e.g., how many years have you been playing a musical instrument, how many years have you been playing computer/console games).

\section{Results for Experiment 1}

We consider the results regarding the effect of music, levels of concentration and the sense of immersion on the remembering of facts in each of the following subsections.

\section{Results for music}

Figure 3 shows the mean number of correctly answered questions from the content section of the questionnaire for each of the experimental conditions along with the standard deviations for the participants.

Descriptive statistics in Figure 3 show the mean scores and standard deviations for each of the five categories of musical manipulations and the control group, as well as for each tempo and pitch condition as aggregate. The mean number of correctly answered questions ranged from a minimum of 14.7 questions by the control group without music (Experiment Condition 10) to a maximum of 17.6 questions by the group under the slow tempo/low pitch condition (Experiment Condition 1). The magnitudes of the mean scores of the musical conditions are close to each other and the variations are wide, therefore it is hard to detect any statistically significant difference between the means (only a difference of 1.7/29 facts between the highest and lowest number of correctly answered questions). Even though the participants who listened to background music during the computer-animated history lesson of the Macquarie Lighthouse performed better than their peers in the no-music control group, a one-way analysis of variance (ANOVA) showed that the experimental (music) conditions were not a significant main effect for learning $(F(5,71)=0.63, p=0.68)$. In other words, we were unable to detect any statistically significant difference between the mean number of correctly answered questions by participants under the six experimental conditions. We also observe from Figure 3 that the slow tempo/low pitch participants are the best performers in terms of learning (mean $=17.6)$.

In Figure 4 we can see the mean number of questions answered correctly was higher under the low pitch condition compared to the high pitch condition. Furthermore, the slow tempo (upper light grey line) had a better effect on learning than the fast tempo (lower dark 


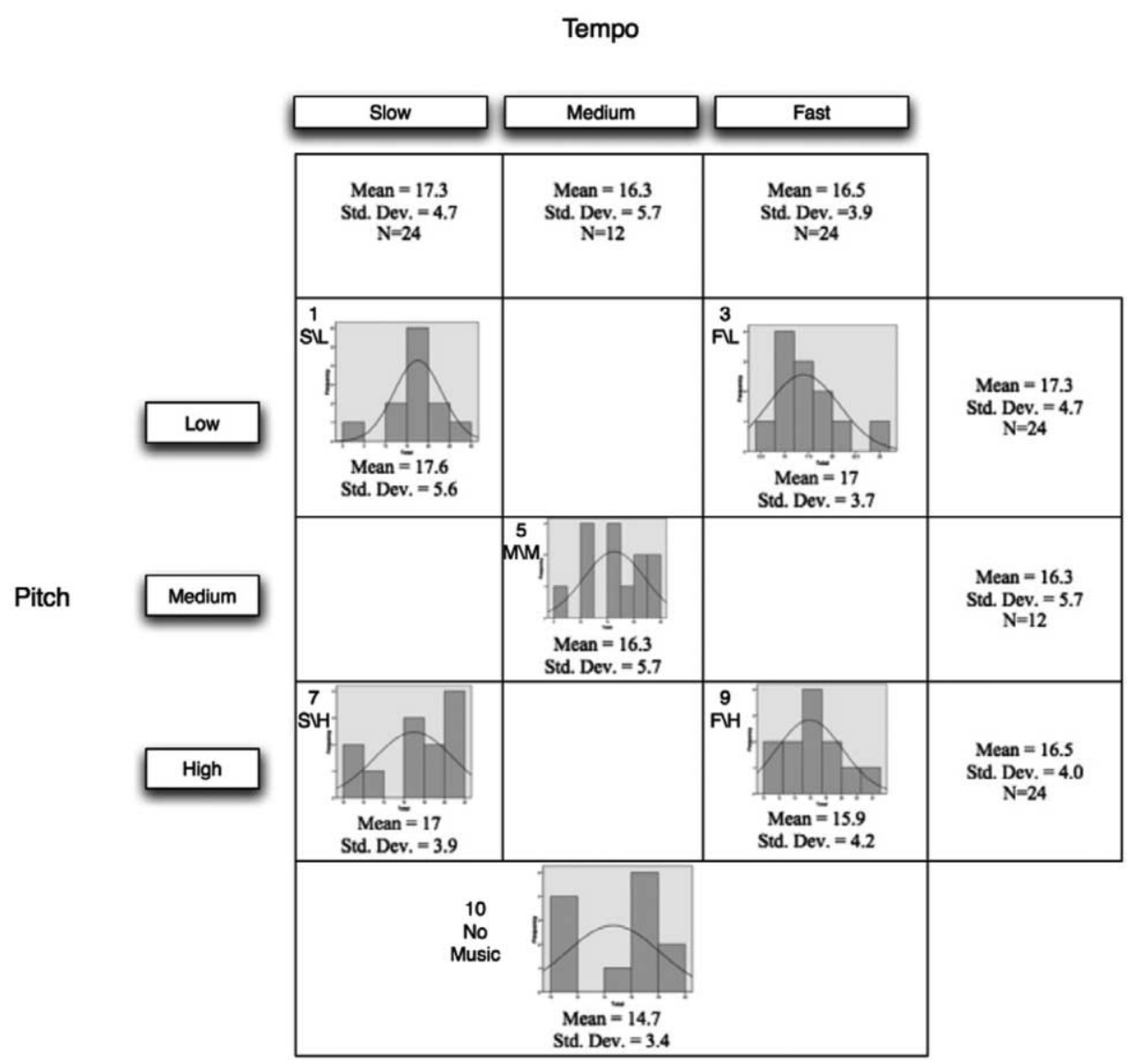

Figure 3. Comparison of the mean number of correctly answered questions in the virtual-immersive $3 \mathrm{D}$ narration. Histograms show the frequencies of correctly answered questions as bars and the normal distribution as a normal curve.

grey line), regardless of the pitch. We investigated the effects of tempo and pitch on learning by using two-way analysis of variance (ANOVA). Neither tempo nor pitch as main effects had a statistically significant effect on learning (both $F(1,45)=0.44, p=0.51$ ). The interaction of these effects was also not statistically significant $(F(1,44)=0.04, p=0.85)$.

Each study participant was exposed to one piece of game music (Oblivion, Baldur's Gate, World of Warcraft, Icewind Dale), except the participants in the control group (no music 12 participants). Each game music was listened to by 15 participants. We compared the effects of different game music on learning regardless of which tempo and/or pitch was used. We found a significant difference between the 'Oblivion' soundtrack and the 'No Music' condition using Dunnett's two-sided t-test $(\mathrm{p}=0.034)$. Those participants who listened to the 'Oblivion' soundtrack, on average, answered 4.4 (95\% confidence intervals $0.3-8.5)$ questions more correctly than their peers in the 'No Music' (control group) (Figure 5).

\section{Results regarding concentration}

A one-way ANOVA contrast test established that music made it subjectively easier to concentrate (Table 1). Participants under the 'Slow Tempo/Low Pitch' condition found it 


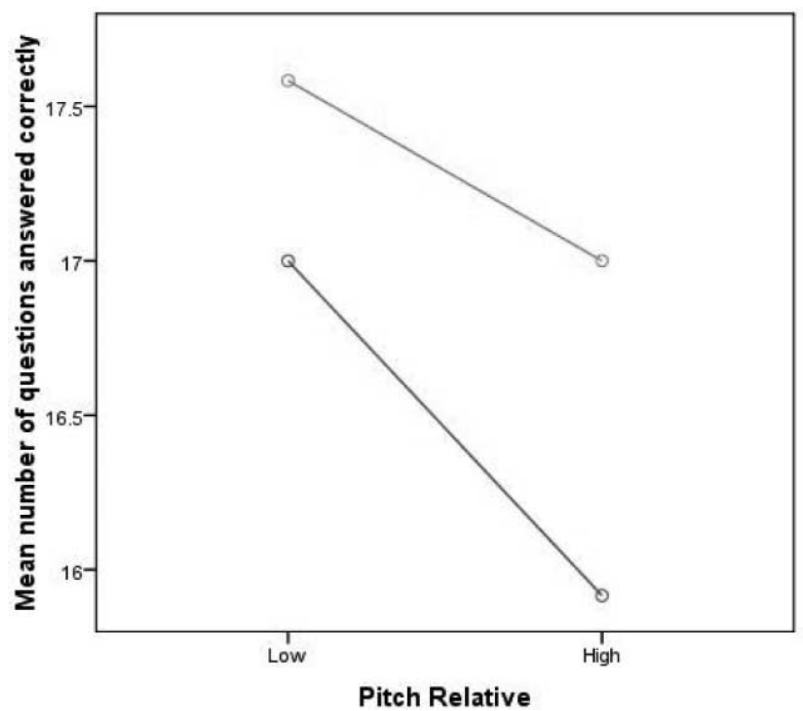

Tempo Relative - Fast

- Slow

Figure 4. The mean number of questions answered correctly by tempo and pitch (original soundtrack condition - medium tempo/medium pitch is removed).

easiest to concentrate. Participants in the control group (no music) found it hardest to concentrate. Participants who did not listen to music found it harder to concentrate with the biggest difference between Slow Tempo/Low Pitch and No Music (Figure 6).

\section{Results regarding immersion}

Moreover, to investigate the feeling of immersion we asked participants a number of related questions including the degree to which they lost track of time in the IVW. They answered on a 5 point scale from 0 having 'Not at all' lost track of time and 4 having 'Completely'

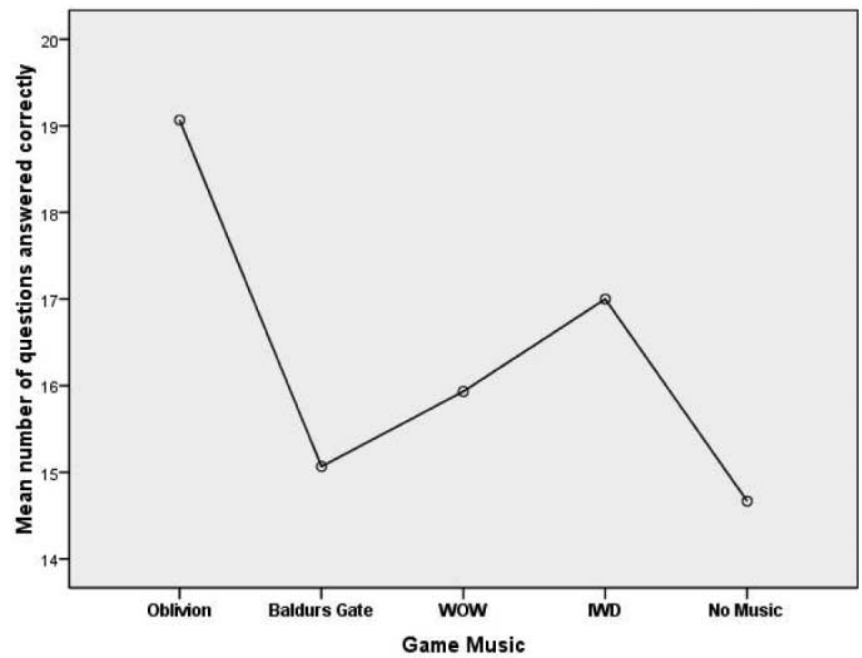

Figure 5. The mean number of questions answered correctly while listening to game music or no music (control). 
Table 1. One-way ANOVA contrast test - Music makes easier to concentrate (subjective selfevaluation of participants).

Contrast Coefficients

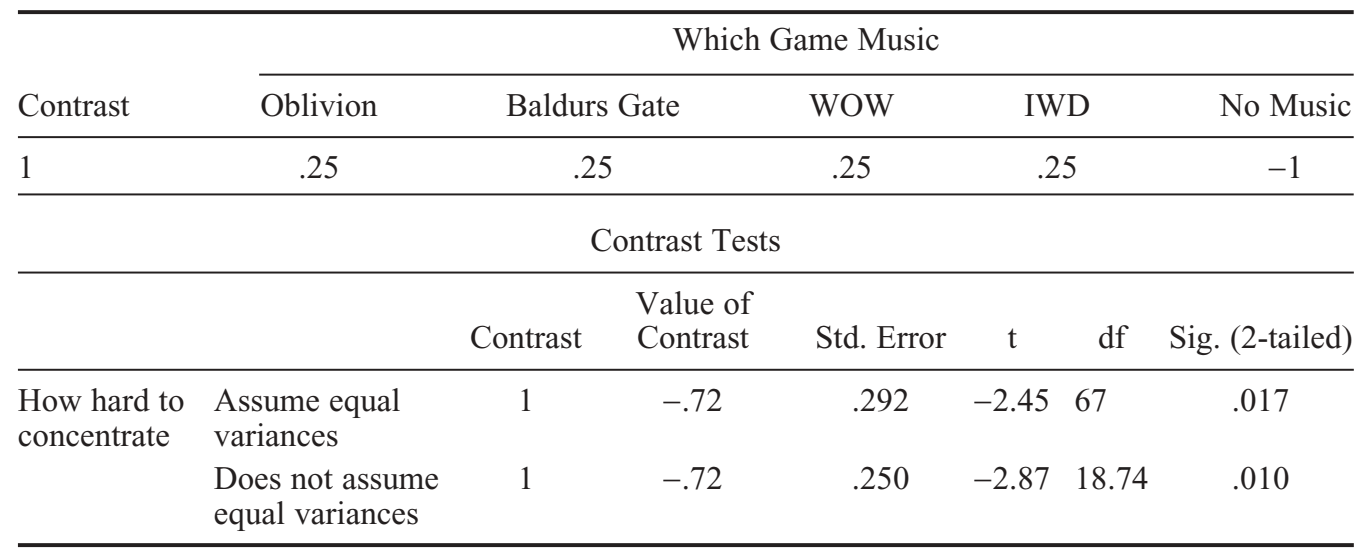

lost track of time. Losing track of time was highly correlated with the total number of questions answered correctly $(\mathrm{r}=0.37, \mathrm{p}=0.003)$. This correlation was statistically significant at 0.01 level. The linear regression showed that $35 \%$ of variation in the total number of questions answered correctly was explained with the degree of losing track of time $\left(\mathrm{R}^{2}\right.$ $=0.347$ ). Based on the linear regression, we conclude that on average if participants did not lose track of time, they answered 11.8 questions correctly. For each further level of losing track of time (i.e. from 1 to 4 on the scale) the total number of questions answered correctly increased by 1.9. For example, if participants completely lost track of time, then on average they would have answered 19.4 questions correctly, which is 7.6 more questions than those

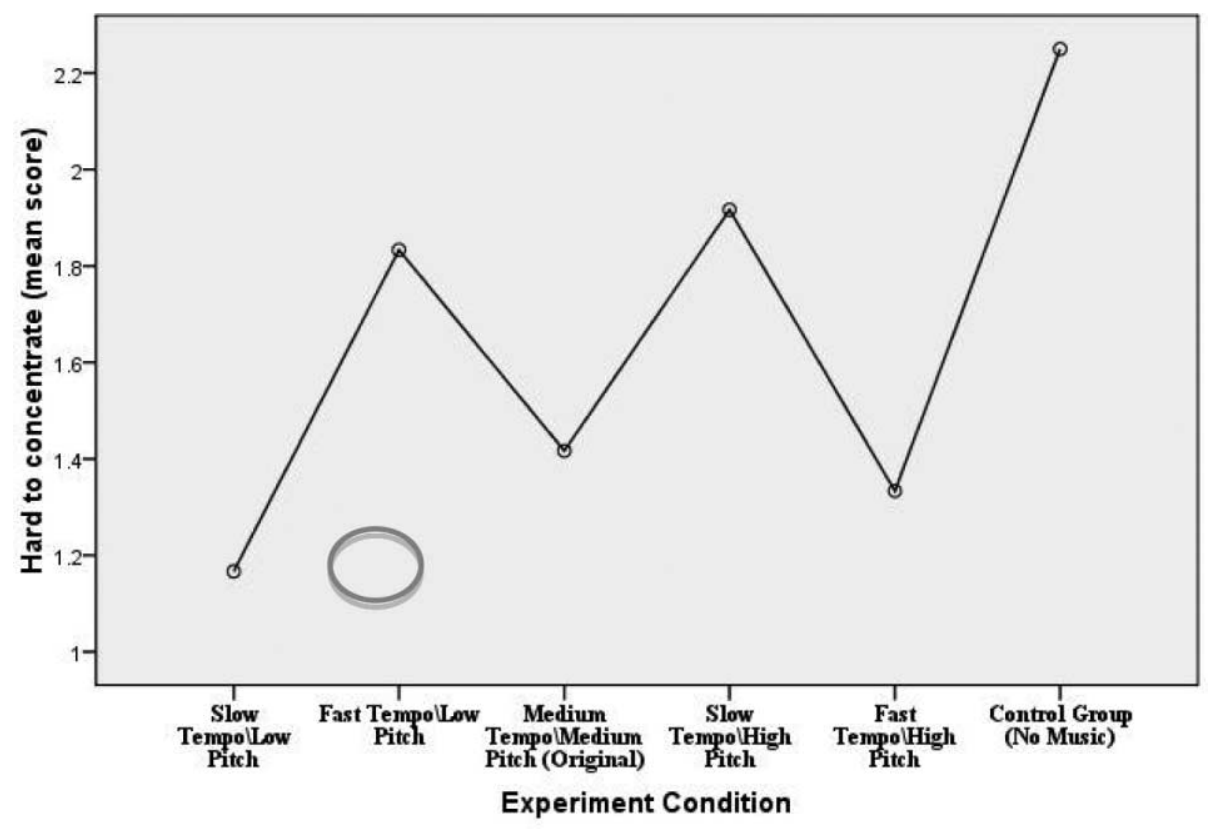

Figure 6. 'How hard was it to concentrate?' under the influence of different musical background stimuli. 
participants who did not lose track of time at all. There was no significant relationship between tempo of the background music and losing track of time.

\section{Experiment 2}

One problem that became obvious during analysis of the data from Experiment 1 was that the absolute tempo and pitch of the various soundtracks differed quite widely from each other. Other problems were the small number of participants in each of the experimental conditions and the 'between subjects' design. These limitations were addressed in Experiment 2. Further, although results were not statistically significant, we were interested in testing the trend in the data showing that the numbers of correctly answered questions are relatively greater with slower tempo and lower pitch. Therefore we decided to narrow our focus by using slow tempo and low pitch experimental conditions. The design of Experiment 2 can be seen in Figure 7. Firstly, the experimental conditions and included variables were reduced to one soundtrack (Oblivion) and a silent condition. These two conditions were then presented to participants in a 'within subjects' design to allow a more direct analysis as opposed to the 'between subjects' design of the first experiment. Thirdly, to eliminate the problems of differing absolute tempo and pitch, only one version of the tempo and pitch manipulations has been used (slow tempo, low pitch which showed beneficial tendencies). While we recognise that this experiment design means that the results might not be generalisable for the whole genre of music of computer role-playing games, this design allows for a more concise analysis. Also, the overall number of participants needed was reduced while increasing the number of participants for each of the new individual conditions. To provide enough statistical power it was decided to include 24 participants in each group, resulting in 48 participants overall.

Group 1 watched the computer-animated history lesson with the Oblivion soundtrack (slow tempo/low pitch) in the first half of the lesson and silence in the second half. Group 2 watched the two conditions in reverse order (silence first, then Oblivion). These parts go for 5:32 (first half) and 5:23 (second half). The slight difference in length is due to a sectional break which could only be made after a sentence was finished by the narratoravatar. While most of the questions from the questionnaire of the first experiment were reused to allow for cross-analysis, a problem in the original experiment was that the number

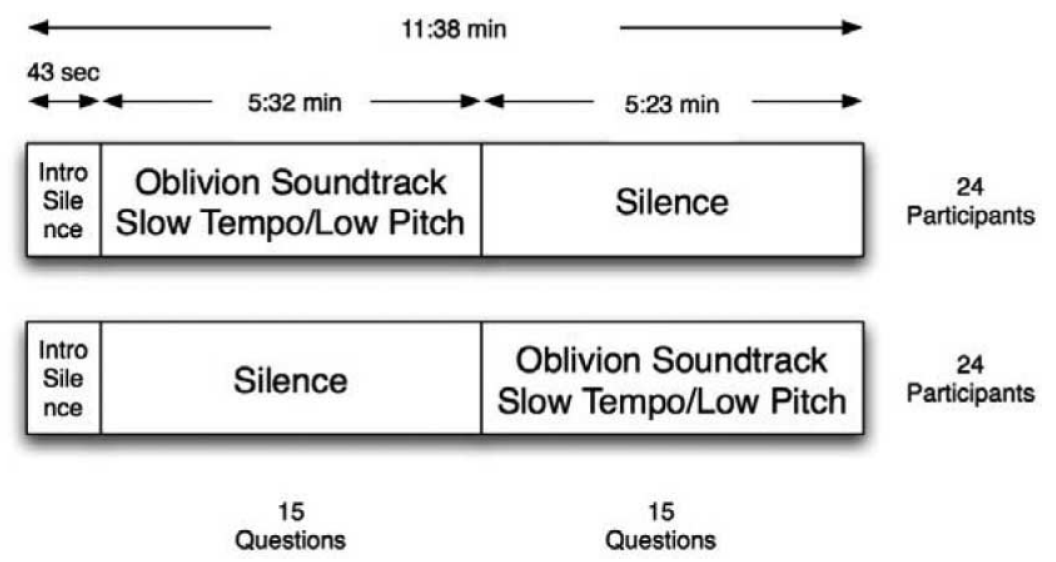

Figure 7. Experiment design for follow-up experiments focuses on one soundtrack and investigates the effect on memory of one particular soundtrack in a 'within subjects' design. 
of facts in the two halves was unbalanced. Thus, four questions were omitted from the first half and five questions added to the second half. This way there are 15 questions in each half (30 overall). The complexity and type of questions in both halves are similar.

Furthermore, some qualitative questions and additional questions regarding immersion and presence were included. In this regard, it was decided to also test the effect of different display systems on feelings of immersion. Thus, half the participants watched the computeranimated history lesson in the Cone display system and the other half was allocated to a 3monitor display system on a computer desk.

\section{Preliminary results for Experiment 2}

The results show that the change from a 'between subjects' design to a 'within subjects' design could not confirm the benefit of the Oblivion soundtrack at slow tempo and low pitch. In other words, we did not observe a direct statistically significant difference between the half where participants listened to background music and the half where they listened only to the narrator without background music. However, the different display systems showed a statistically significant difference $(p=.032)$ in favour of the 3-monitor solution. Participants in the Cone system remembered 14.96 items on average and 17.96 items in the 3 -monitor display system. These results then led to closer investigations which revealed a three-way interaction between the display system, the music/no-music condition and the two halves of the history lesson. Interestingly, when the Cone and 3-monitor display groups were separated and the music condition was then compared with the no-music condition within these groups, it was discovered that those participants in the 3-monitor solution who listened to music in the first half of the computer-animated history lesson remembered significantly more facts from the second half than their peers who listened to no music in the first half. In contrast, those participants in the Cone system performed significantly better if they listened to no music in the first half and music in the second half. These results are statistically significant at .027 (3-monitor) and .017 (Cone) level. A possible explanation for this result is that participants in the Cone system experienced cognitive overload when faced with the overwhelming experience of an unfamiliar display system together with the music. One observation by the experimenters from this second experiment is that participants seemed to feel much more comfortable with the 3-monitor solution than in the Cone system. However, analysis and interpretation of these results are ongoing.

\section{General discussion}

In summary, changes in tempo and pitch did not show a statistically significant difference on the amount of correctly answered questions. Nor did 'Music' versus 'No Music' if all soundtracks were combined in the same (Music) category. However, when we looked at each individual soundtrack and compared it to the 'No Music' control group in the first experiment, we found a statistically significant difference between the Oblivion soundtrack and the control group. The statistically significant result for the Oblivion soundtrack indicates that it may be beneficial for encoding information into memory. When we consider what distinguishes Oblivion from the other samples, we are not able to pinpoint tempo or pitch.

One conjecture is that Oblivion could be the most congruent piece for the setting of the lighthouse scene. The importance of congruency between music and accompanying media has been the subject of considerable research. Properties of music that are congruent with accompanying media act to highlight certain features of that media over others and hence 
can greatly influence how those media are remembered. In advertisements for cars, for example, accompanying classical music can act to highly convey both the vitality and high cultural associations of the car (Cohen 2001). As another example, music that exhibits an unambiguous ending may connote the end of a film (Thompson, Russo, and Sinclair 1994). According to the congruence-associationist model outlined by Marshall and Cohen (1988), sources of congruency act by directing attention to particular aspects of an accompanying film over others (see also Bolivar, Cohen, and Fentress 1994). These effects on attention, in turn, influence interpretations and memory for that material.

Finally, we found a statistically significant effect between the number of questions answered correctly and the participant's feeling of immersion in the virtual-immersive environment. Those who said that they were more immersed (by saying that they lost track of time) in the 3D history course remembered more facts than those who said that they did not lose track of time. Concentration was also positively affected in the music conditions.

\section{Conclusions}

The role of background music in games and IVWs is a largely unexplored field. Previous studies concerning music and learning or intelligence have had mixed results and as in the case of the Mozart effect, it is difficult to repeat findings. In our own studies we did find one soundtrack which showed a statistically significant improvement in memorisation of facts. We conjectured that the reason may be that the piece of music was most congruent to the content being taught, but clearly more research needs to be conducted in this area. We also found a link between levels of perceived immersion and ability to accurately remember facts. Again the link may not be direct between music, memory and the sense of immersion, but the link is worth investigating further. Further analysis of the data from the second experiment is being conducted to this end. It is our goal to take the field of IVW beyond the intuitions of gamers and game developers and to contribute towards unpacking the appeal and benefits of IVWs as a learning environment and the role that music may play within that environment.

\section{Acknowledgements}

Thanks to Alan Taylor for his contribution, his general interest in this work and particularly for his data analyses related to the experiments. This project is partly funded by Australian Research Council Discovery Grant coded DP0558852 and Macquarie University Research Infrastructure Grant titled 'Virtual Reality Engine'. The principal author is funded by iMURS (international Macquarie University Research Scholarship).

\section{References}

Bolivar, V.J., A.J. Cohen, and J.C. Fentress. 1994. Semantic and formal congruency in music and motion pictures: Effects on the interpretation of visual action. Psychomusicology 13, no. 1: 28-59.

Boltz, M.G. 2004. The cognitive processing of film and musical soundtracks. Memory \& Cognition 32, no. 7: 1194-205.

Boltz, M.G., M. Schulkind, and S. Kantra. 1991. Effects of background music on the remembering of filmed events. Memory \& Cognition 19, no. 6: 593-606.

Chen, J. 2007. Flow in games (and everything else). Communications of the ACM 50, no. 4: 31-4.

Cohen, A. 2001. Music as a source of emotion in film. In Music and emotion: Theory and research, ed. P. Juslin and J. Sloboda, 249-72. Oxford: Oxford University Press.

Csikszentmihalyi, M. 1990. Flow: The psychology of optimal experience. New York: Harper \& Row. 
Davies, M.A. 2000. Learning ... The beat goes on. Childhood Education 76, no. 3: 148.

Draper, S.W., J. Cargill, and Q. Cutts. 2002. Electronically enhanced classroom interaction. Australian Journal of Educational Technology 18, no. 1: 13-23.

Dryden, G., and J. Vos. 2001. The learning revolution - to change the way the world learns. Stafford: Network Educational Press Ltd.

Eady, I., and J.D. Wilson. 2004. The influence of music on core learning. Education 125, no. 2: 243-8.

Fassbender, E., and W. Heiden. 2006. The virtual memory palace. Journal of Computational Information Systems 2, no. 1: 457-64.

Holt, R. 2000. Examining video game immersion as a flow state. B.A. Thesis, Department of Psychology, Brock University, St. Catharines, Ontario, Canada.

Husain, G., W.F. Thompson, and E.G. Schellenberg. 2002. Effects of musical tempo and mode on arousal, mood, and spatial abilities: Re-examination of the 'Mozart effect'. Music Perception 20, no. 20: 151-71.

Ilie, G., and W.F. Thompson. 2006. A comparison of acoustic cues in music and speech for three dimensions of affect. Music Perception 23, no. 4: 319-29.

Landauer, D. 1995. The trouble with computers: Usefulness, usability, and productivity. Cambridge, MA: MIT Press.

Lozanov, G. 1988. The foreign language teacher's suggestopedic manual. New York: Gordon and Breach Science Publishers.

- 1992. Suggestology and outlines of suggestopedy. Philadelphia: Gordon and Breach Science Publishers.

Marshall, S.K., and A.J. Cohen. 1988. Effects of musical soundtracks on attitudes toward animated geometric figures. Music Perception 6: 95-112.

Mason, R.O., and I.I. Mittroff. 1973. A program for research on management information systems. Management Science 19, no. 5: 475-87.

McFarland, R.A., and R.F. Kennison. 1988. Asymmetrical effects of music upon spatial-sequential learning. Journal of General Psychology 115, no. 3: 263.

McKelvie, P., and J. Low. 2002. Listening to Mozart does not improve children's spatial ability: Final curtains for the Mozart effect. British Journal of Developmental Psychology 20, no. 2: 241.

Rauscher, F.H., G.L. Shaw, and K.N. Ky. 1993. Music and spatial task performance. Nature 365, no. 6447: 611 .

Richards, D. 2006. Is interactivity actually important? Proceedings of the Third Australasian Conference on Interactive Entertainment (IE'2006). Perth: Murdoch University.

Rideout, B.E., and S. Dougherty. 1998. Effect of music on spatial performance: A test of generality. Perceptual \& Motor Skills 86, no. 2: 512.

St. Julien, T.U., and C.D. Shaw. 2003. Firefighter command training virtual environment. In Proceedings of the 2003 Conference on Diversity in Computing, Atlanta, Georgia, USA, October 15-18, 2003, TAPIA '03, 30-33. New York: ACM.

Thompson, W.F., F.A. Russo, and D. Sinclair. 1994. Effects of underscoring on the perception of closure in filmed events. Psychomusicology 13: 9-27.

Thompson, W.F., E.G. Schellenberg, and G. Husain. 2001. Arousal, mood, and the Mozart effect. Psychological Science 12, no. 3: 248-51.

Vester, F. 1975. Denken, lernen, vergessen : Was geht in unserem Kopf vor, wie lernt das Gehirn, und wann lässt es uns im Stich? Stuttgart: Deutsche Verlags-Anstalt.

Weisskoff, R.S. 1981. The relationship of pop/rock music to children's task performance and continuing motivation in language arts instruction. Hartford, CT: University of Connecticut.

Zehnder, S.M., and S.D. Lipscomb. 2006. The role of music in video games. In Playing Video Games: Motives, Responses, and Consequences, ed. P. Vorderer and J. Bryant, 241-7. Mawwah, NJ: Lawrence Erlbaum Associates Publishers. 